Michaela Christine Hastetter

Albert-Ludwigs-Universität Freiburg

\title{
Das neue Lied singen...
}

\section{Zur Kunst des Aggiornamento}

\section{Hinführung}

In der dritten Auflage des Lexikons für Theologie und Kirche umschreibt Giuseppe Alberigo „Aggiornamento“ als die Suche nach einer „neuen Inkulturation der Offenbarung [...] in einer Menschheit, die im Umbruch begriffen ist ${ }^{\text {" }}{ }^{1}$ Welche Spannung sich dabei auftut, sei aus der Distanz einer anderen Disziplin an einem verwandten Phänomen erläutert. 1993 trat der Freiburger Dirigent und Komponist Hans Zender mit einem neuen Werk an die Öffentlichkeit, das folgenden Titel trägt: Schuberts "Winterreise" - Eine kompositorische Interpretation für Tenor und kleines Orchester, das mittlerweile zu den meist verkauften Einspielungen aus dem Bereich der Neuen Musik zählt. ${ }^{2}$ Er selbst gibt relativ genaue Auskunft über seine künstlerische Intention: „Seit der Erfindung der Notenschrift ist die Überlieferung von Musik geteilt in den vom Komponisten fixierten Text und die vom Interpreten aktualisierte klingende Realität" ${ }^{3}$ Als Dirigent

\footnotetext{
G. Alberigo, Aggiornamento, [in:] ${ }^{3}$ LThK 1, 231.

So die Auskunft beim Kauf der Aufnahme in der CD-Abteilung der Buchhandlung Rombach in Freiburg im Breisgau.

H. Zender, Notizen zu meiner Bearbeitung der „Winterreise“, in: Booklet zur CD Hans Zender, Schuberts "Winterreise“. Eine komponierte Interpretation. Christoph Prégardien. Klangforum Wien. Sylvian Cambreling, Kairos Music Production 1999, 4-7: 4 (woraus im Folgenden zitiert wird); be-
} 
hat sich Zender zeitlebens mit der Interpretation von Meisterwerken aus der Musikgeschichte bemüht. Für ihn ist der Notentext jedoch keine eindeutige „Beschreibung von Klängen“, sondern vielmehr „Aufforderung zur Aktion“: „Es bedarf des schöpferischen Einsatzes des Interpretierenden, seines Temperamentes, seiner Intelligenz, seiner durch die Ästhetik der eigenen Zeit entwickelten Sensibilität, um eine wirklich lebendige und erregende Aufführung zustande zu bringen ". ${ }^{4}$ Für Zender wird dabei der Interpret zum Mitautor, sein Tun „schöpferische Veränderung“. ${ }^{5}$ Was für den Dirigenten Zender im Grunde Konstitutivum jeder neuen Einstudierung und Aufführung ist, hat Zender als Komponist an seiner „kompositorischen Interpretation“ von Schuberts Winterreise nun ad exemplum vorgeführt: Aus verschiedenen Perspektiven liest er den Text neu, verfremdet, interpretiert. ${ }^{6}$ Seine kompositorischen Eingriffe haben zum Ziel, alte Hörgewohnheiten aufzubrechen, die zukunftsweisende Dynamik des Originals herauszuarbeiten, um letztlich „die existentielle Wucht des Originals neu zu erleben".7

Der interpretatorischen Verfremdung stellt Zender einen zweiten Umgang mit Schuberts Original gegenüber, der auf eine andere Weise das Gewöhnliche und Allzubekannte durchbricht, seiner Version von Verheutigung jedoch völlig entgegengesetzt ist: Es ist die Pflege des sogenannten „heilige[n] Original[s] [...] auf Hammerklavieren, SchubertFlügeln, Kurzhalsgeigen und Holzflöten “. ${ }^{8}$ Diese mit dem Namen Nikolaus

reits abgedruckt in der vom Verlag Breitkopf \& Härtel herausgegebenen Zeitschrift: „Up To Date“ 3 (1993) 2-5.

4 H. Zender, Notizen, 4.

Ebd.

6 Zender wörtlich: „Meine «lecture» der Winterreise sucht nach einer neuen expressiven Deutung, sondern macht systematisch von Freiheiten Gebrauch, welche alle Interpreten sich normalerweise auf intuitive Weise zubilligen“ (ebd., 5). Darunter fallen für ihn Dehnung bzw. Raffung des Tempos, Transposition in andere Tonarten, Springen innerhalb des Textes, Mehrfachwiederholungen, Unterbrechung der Kontinuität, Permutation von Klangfarben, Hinzufügen von frei erfundenen Klängen (Kontrafakturen), Vor-, Nach- oder Zwischenspiele.

H. Zender, Notizen (wie Anm. 3), 7; zur gesamten Auseinandersetzung mit Zenders „Winterreise“ vgl. auch K. H. Stahmer, Bearbeitung als Interpretation. Zur Schubertrezeption Gustav Mahlers, Hans Zenders und Friedhelm Döhls, in: ders. (Hg.), Franz Schuber und Gustav Mahler in der Musik der Gegenwart („Schriften der Hochschule für Musik Würzburg“ 5), Mainz u.a. 1997, 25-61, zu Zender 44-55.

\footnotetext{
8 H. Zender, Notizen (wie Anm. 3), 5.
} 
Harnoncourt eng verbundene historische Aufführungspraxis, hat das Ziel, den historischen Originalklang eines Werkes durch die Verwendung von Instrumenten und Spieltechnik aus der Entstehungszeit möglichst originalgetreu zu reproduzieren. ${ }^{9}$ Für Zender taugt sie nur insofern, als mit ihr liebgewonnene Hörgewohnheiten in Frage gestellt werden, „um den heutigen Hörer zu verunsichern". ${ }^{10}$ Selbst Harnoncourt sieht im Ansinnen, historische Musik „nicht in die Gegenwart hineinzuholen, sondern sich selbst in die Vergangenheit zurückzuversetzen" letztlich eine Verfallserscheinung und das „Symptom des Verlusts einer wirklich lebendigen Gegenwartsmusik“.11

9 Zur Definition von werkgetreuer oder historischer Aufführungspraxis: „Werkgetreu ist eine Wiedergabe dann, wenn sie sich der Vorstellung des Komponisten zur Zeit der Komposition annähert" (N. Harnoncourt, Musik als Klangrede. Wege zu einem neuen Musikverständnis, München u.a. $\left.{ }^{3} 1987,15\right)$. Hierzu der Problemkomplex, den Harnoncourt mit der Frage nach der historischen Aufführung verbindet: „Die Musik der Vergangenheit ist durch den Lauf der Geschichte, durch die Entfernung von der Gegenwart, durch das Losgerissensein von der eigenen Zeit zu einer Fremdsprache geworden. Einzelne Aspekte mögen allgemein gültig und zeitlos sein, die Aussage als solche ist aber zeitgebunden und kann nur wiedergefunden werden, wenn man sie in eine Art von Übersetzung in die heutige Zeit hinein bringt. Das heißt: Wenn die Musik vergangener Epochen überhaupt noch in einem tieferen und weiteren Sinn für die Gegenwart aktuell ist, wenn sie in ihrer ganzen Aussage [...] dargestellt werden soll, muß das Verständnis dieser Musik von ihrer Gesetzmäßigkeit her wieder erlernt werden. Wir müssen wissen, was die Musik sagen will, um zu erkennen, was wir mit ihr sagen wollen. Also muß jetzt zum rein Gefühlsmäßigen, zur Intuition, das Wissen kommen. Ohne dieses historische Wissen kann historische Musik, kann unsere sogenannte «Ernste Musik» nicht adäquat dargestellt werden“ (ebd., 25f.).

10 So die Einschätzung zu Zenders abwehrender Haltung gegenüber der historischen Aufführungspraxis von K. H. Stahmer, Bearbeitung als Interpretation (wie Anm. 7), 44.

11 N. Harnoncourt, Musik als Klangrede (wie Anm. 9), 14, hier weiter: „Die Musik von heute genügt weder dem Musiker noch dem Publikum, ja der größte Teil lehnt sie direkt ab, und um das so entstehende Vakuum auszufüllen, greift man auf historische Musik zurück“ (ebd.). Andererseits kann Harnoncourt dieser Entwicklung auch etwas Positives abgewinnen: „Die historische Einstellung hat auch ein Gutes: sie ermöglicht uns, erstmalig in der Geschichte unserer christlich-abendländischen Kunst, einen freien Standpunkt einzunehmen und so das ganze Schaffen der Vergangenheit zu überblicken“ (ebd., 15). Und später: „Ich möchte hier absolut nicht für «historische» Aufführungen, für Rekonstruktionen von Aufführungen früherer Zeiten, plädieren, das Rad der Geschichte kann nicht zurückgedreht werden. Aber bei aller Progressivität brauchen wir offenbar doch die Kunst, die Musik vergangener Zeiten, das Klanggewand ist und bleibt eine sekundäre Sache. Für mich ist das originäre Klangbild nur insofern interessant, als es mir unter den vielen, mir zur Verfügung stehenden Möglichkeiten die beste zu sein scheint, diese oder jene Musik heute darzustellen“ (ebd., 101). 


\section{Aggiornamento-Verständnis im Wandel}

Die beiden Ansätze - Zenders interpretatorische Neukomposition im Spannungsfeld von überliefertem Text und aktualisierter Realität und Harnoncourts historische Aufführungspraxis von Musik als Rückbesinnung auf ihre Entstehungszeit haben uns ins Zentrum unserer pastoraltheologischen Fragestellung zum Aggiornamento, dem gelingenden Zueinander von Überlieferung und Heute, Beständigkeit und Wandel, geführt. Pastoraltheologisch ist die Frage nach Michael Bredeck deshalb, weil die Erneuerung der Kirche nicht aus ,abstrakt gewonnenen theologischen Prinzipien abgeleitet" wird, sondern sich aus dem „lebendigen Zusammenspiel von Evangelium und gegenwärtiger Geschichte" ${ }^{\prime 12}$ ergibt. Wie der Dirigent steht auch der Seelsorger vor der Herausforderung der Vergegenwärtigung des christlichen Glaubens, der Übersetzung eines Überkommenen in die Gegenwart. Doch welcher Weg ist einzuschlagen - interpretatorische Neukomposition oder historisierende Rückschau? Um sich hierüber Klarheit zu verschaffen, lohnt es sich, an die Quellen zurückzukehren. Denn obwohl die Einführung des Paradigmas „Aggiornamento“ in die theologische Denkwelt kaum 60 Jahre zurückliegt, unterliegt der Begriff selbst schon wieder einem Verständniswandel, der kurz skizziert werden soll.

\subsection{Aggiornamento-Verständnis zu Beginn des Konzils}

Wegweisend wurde Aggiornamento bekanntlich durch Johannes XXIII., der dieses Leitwort, das schon seine „Scritti e Discorsi“ als Patriarch in Venedig durchzog, ${ }^{13}$ in seine Ansprache zur Eröffnung des Zweiten Vatikanischen Konzils am 11. Oktober 1962 aufnahm. Hierin gibt er Auskunft darüber,

12 M. Bredeck, Das Zweite Vatikanische Konzil des Aggiornamento. Zur hermeneutischen Grundlegung einer theologischen Konzilsinterpretation (Paderborner Theologische Studien 48), Paderborn u.a. 2007, 27.

13 Vgl. dazu die Auswertung von A. G. Roncalli, Patriarca di Venezia. Scritti e Discorsi, 3 Bde, I: 1953-1954, Roma 1959; II: 1955-1956, Roma 1959; III: 1957-1958, Roma 1959 bei Bredeck, Zweite Vatikanum (wie Anm. 12), 195-237; zur Entstehung und Gestalt des Aggiornamento-Verständnisses bei Angelo Roncalli - Papst Johannes XXIII. vgl. ausführlich F. M. Willam, Vom jungen Angelo Roncalli (1903-1907) zum Papst Johannes XXIII. (1958-1963). Eine Darlegung vom Werden des Aggiornamento-Begriffs 1903-1907 als der Leitidee für das II. Vatikanische Konzil und die Durchführung seiner Beschlüsse - ein aktuelles Buch, Innsbruck 1967. 
in welchem Sinne er Aggiornamento versteht. ${ }^{14}$ Auffallend ist hierbei die Spannung zwischen Kontinuität und Vitalität, zwischen der Überlieferung und dem Heute, zwischen wirksamerem Bewahren (custodire) und Vorlegen (proponere), die sich gewissermaßen durch den gesamten Text zieht und gleichsam den hermeneutischen Schlüssel für das Aggiornieren bildet. ${ }^{15}$ Seine Vision des Aggiornamento hat demnach zweierlei im Blick: das unveräußerliche Glaubensgut, das die Kirche aus der Vergangenheit empfangen hat und jenes Achten auf die Gegenwart, ,auf die neuen Lebensverhältnisse und -formen, wie sie durch die moderne Welt geschaffen wurden ", ${ }^{16}$ auf das Heute also, dem die Weitergabe des Überlieferten entsprechen müsse. Aus seinen Worten spricht keine historische Aufführungspraxis, und ebenso wenig ein „nachschöpferische[r] Eingriff“, mit dem ein „,neues Original“"17 entsteht. Für ihn liegt die Kunst der Seelsorge im schwierigen Gleichgewicht eines et...et, eines sowohl...als auch, das eine Mitte zwischen beiden Extremen findet. Diese Kunst vermeidet die ausschließliche Anpassung der Kirche an den Zeitgeist ebenso wie museale Spiritualität, die den Bezug zum zeitbedingten Kontext verliert. ${ }^{18}$ Franz Michel Willam hat in diesem Kontext darauf hingewiesen, dass es Johannes XXIII. gelun-

14 Vgl.hierzu Johannes XXIII., Gautet Mater Ecclesia. Ansprache zur Eröffnung des Zweiten Vatikanischen Konzils (11.Oktober 1962), in: J. Kaufmann; N. Klein, Johannes XXIII. Prophetie und Vermächtnis, FribourgBrig 1990, 116-149, darin schon in Nr. 2: „,...] Vitalität der katholischen Kirche (vigorem Ecclesiae /vitalità) [...] die Kontinuität des kirchlichen Lehramtes (Magisterium Ecclesiasticum, numquam deficiens et ad finem usque temporum perseveran / continuità)“ (ebd., 117). Zur Übersetzungsproblematik der Eröffnungsansprache des Papstes vgl. L. Kaufmann; Johannes XXIII. Prophetie im Vermächtnis, Hg. von N. Klein, Fribourg-Brig 1990, 107-115 mit weiterführenden Literaturhinweisen zur Textgenese und Synopse der verschiedenen Textfassungen und Übersetzungen.

15 Als Hauptaufgabe des Konzils sieht der Papst, „das unveräußerliche Überlieferungsgut der christlichen Lehre (sacrum christianae doctrinae depositum) wirksamer zu bewahren (custodiatur) und zu lehren (wörtl. vorzuschlagen/proponeatur)" (Johannes XXIII., Gaudet Mater Ecclesia [wie Anm. 14], Nr. 11).

16 Ebd., Nr. 12, und ein wenig weiter unten im Text: „Unsere Aufgabe ist es nicht nur, diesen kostbaren Schatz zu bewahren, als ob wir uns nur um Altertümer kümmern würden. Sondern wir wollen uns mit Eifer und Furcht der Aufgabe widmen, die unsere Zeit fordert“" (ebd., Nr. 14).

17 K. H. Stahmer, Bearbeitung als Interpretation (wie Anm. 7), 44.

18 Bredeck kann hier zugestimmt werden, wenn er „gegen beide tendenziell ideologischen Verständnisansätze des Aggiornamento gerade die Verknüpfung von Glaubensvertiefung und Zeitbezug geltend zu machen“ (M. Bredeck, Zweite Vatikanum [wie Anm. 12], 29) versucht; zur Kunst des Aggiornierens vgl. F. M. Willam, Vom jungen Angelo (wie Anm. 13), 146-148. 
gen ist, das Prinzip der theologischen Anpassung, ${ }^{19}$ welches er von John Henry Newman übernommen habe, mit der Substanz-Akzidenz-Lehre des Thomas von Aquin zu verbinden: „Die theologische Anpassung vollzieht sich auf dem Wege, daß die Substanz der Lehre beim Wechsel von einem Zeitalter zum anderen beibehalten wird, die Akkzidentien, d.h. die Darlegungen aber, die dem abgelaufenen Zeitalter entsprechen, nun durch solche ersetzt werden, die dem neuen entgegenkommen". ${ }^{20}$

19 Die theologische Anpassung macht Willam fest an der Tagebucheintragung vom 16. Januar 1903, in der Roncalli schreibt: „Von der Tugend der Heiligen muß ich die Substan z (U.V.) [sc. wörtlich: das Wesentliche] und nicht die A kz i d e nti en (U.V.) [sc. wörtlich: das Zufällige] übernehmen. Ich bin nicht der hl. Aloisius. Ich muß mich nicht auf die gleiche Weise heiligen, wi e mein von ihm verschiedenes Wesen, mein Charakter, meine von den seinen verschiedenen Lebensbedingungen es bringen. Ich habe nicht die magere und steckendürre Reproduktion eines andenfalls noch so vollendeten Heiligentyps zu sein. (U.V.) Gott will, daß wir in Nachahmung der Beispiele der Heiligen den lebendigen Saft - il succo vitale - ihrer Tugenden in uns einsaugen, ihn in unser Blut umwandeln und den uns eigenen Veranlagungen und besonderen Lebensumständen anpassen - adattare. Wäre der hl. Aloisius jener, der ich bin, würde er auf einem anderen Wege heilig werden, als auf jenem, den er eingeschlagen hat" (Johannes XXIII., Geistliches Tagebuch, zit. hier in der deutschen Übersetzung nach F. M. Willam, Vom jungen Angelo [wie Anm. 13], 32f.). Diese theologische Anpassung oder auch anpassende Interpretation grenzt Willam mit Jan Hendrik Walgrave gegen zwei andere Strömungen ab: das Prinzip der logischen Anpassung, das man zu Beginn des 20. Jahrhunderts als das einzig gültige betrachtete und nach dem nur sprachliche, durch logische Denkprozesse gesicherte Anpassungen der Lehre erlaubt sind, und das Prinzip der modernistischen oder transformistischen Anpassung, ebenfalls um 1900, mit dem nur der religiöse Sinn bleibende Substanz ist, nicht aber die Dogmen, das sie nur zeitgeschichtlich bedingte Formulierungen dieses religiösen Sinnes sind und daher kommen und gehen. Die theologische Anpassung hingegen, die auf Newman zurückgeht, ,gründet auf der Tatsache, daß die Offenbarung von Gott ergangen [sc. ist] und ihre Weitergabe innerhalb der Geschichte der Menschheit und damit auch innerhalb der Tradition unter dem Einfluß hl. Geistes erfolgt“ (F. M. Willam, Vom jungen Angelo [wie Anm. 13], 10); Willam stützt sich hierbei auf das Vorwort von Walgrave zur französischen Übersetzung der „Abhandlung über die Entwicklung der christlichen Lehre“, vgl. J. H. Newman, Essai sur le developpement de la doctrine chrétienne, Traduit de l'anglais par Luce Gerade, Introduction par Jan Hendrik Walgrave, Paris 1964, 29-48.

20 F. M. Willam, Vom jungen Angelo (wie Anm. 13), 146, hier weiter: „In diesem Sinne gebraucht Papst Johannes XXIII. in einer Konzilsansprache den bildlichen Ausdruck «Neubekleidung - rivestimento» der Substanz“ (ebd. mit Verweis auf DMC. 5/13). Diese Herausforderung hat Benedikt XVI. in seiner Ansprache an das Kardinalskollegium und die Mitglieder der römischen Kurie am 22. Dezember 2005 aufgegriffen und unter dem Doppelaspekt von Dynamik und Treue, Diskontinuität und Kontinuität weitergeführt. Dabei unterscheidet er zwischen Entscheidungen der Kirche, die zu ihrem Wesen gehören und daher unveränderlich sind und solchen, die vorübergehend sind und nicht ihr Wesen betreffen. Diese nicht auf das Wesen der Kirche bezogenen Fragen zögen notwendigerweise auch vorübergehende Antworten nach sich, da sie sich auf eine in sich selbst ver- 


\subsection{Das Ringen um das Aggiornamento-Verständnis gegen Ende des Konzils}

Folgt man dem weiteren Verlauf des Konzils, macht sich bereits in seiner Endphase ein Wandel in der Aggiornamento-Rezeption breit, wie aus einem Brief von Henri de Lubac aus dem Jahr 1964 hervorgeht. Er schreibt: „Reform, aggiornamento, Weltoffenheit, [...] usw.: all das muß, im Glauben, als heutige Forderung eines geläuterten und vertieften christlichen Geistes verstanden werden. Doch, derart entstellt, entspricht es in der Vorstellung vieler ungefähr einem Fahrenlassen, [...] ja fast einem Aufgeben von Glauben und Sittlichkeit“. ${ }^{21}$ Dem „wahren aggiornamento" stellt de Lubac deshalb einen anderen Begriff gegenüber, den der „Mutation“22 - eine Abänderung also, bei der das vormals Bestehende in ein anderes, neues Stadium übergeht, das mit dem ersten nicht mehr viel gemeinsam hat. ${ }^{23}$ De Lubac charakterisiert diese schon während des Konzils vorhandene Strömung als ein dem Konzil völlig entgegenstehendes Programm, das in den folgenden Jahren noch weiter um sich greifen sollte. Am 26. Juli 1966 schreibt er an den Redaktor von La Croix, P. Wenger:

änderbare Wirklichkeit beziehen. Bei solchen Entscheidungen seien nur die Grundsätze unveränderlich, während die konkreten Umstände der Veränderung unterworfen sind. Daher sei die Natur wahrer Reform das Zusammenspiel von Kontinuität und Diskontinuität auf verschiedenen Ebenen, vgl. hierzu Benedikt XVI., Ansprache an das Kardinalskollegium und die Mitglieder der Römischen Kurie beim Weihnachtsempfang (22. Dezember 2005) (VApSt 172), Bonn 2006.

${ }_{21}$ H. de Lubac, Annexe [a] Brief an einen Kardinal vom 18. Oktober 1964, in: ders., Meine Schriften im Rückblick. Mit einem Vorwort von Erzbischof Christoph Schönborn (Theologia Romanica 21), Einsiedeln 1996, 439-442: 439, ausgelassen: „einem Indifferentismus oder rückgratlosem Liberalismus, an den "Geist dieser Welt» gemachter Zugeständnisse“. Dass de Lubacs Hauptanliegen in diesem Brief um das rechte Verständnis von Aggiornamento kreist, geht nicht zuletzt aus der Begründung der Wahl des Adressaten hervor: „Die so eindeutige und gerade Haltung, die Ihre Eminenz zugunsten des wahren aggiornamento eingenommen haben, ließ mich Sie als Empfänger wählen“ (ebd., 441).

22 H. de Lubac, Annexe [b], aus: Chemin spirituel de notre temps, 755-771, in: ders., Meine Schriften im Rückblick. Mit einem Vorwort von Erzbischof Christoph Schönborn (Theologia Romanica 21), Einsiedeln 1996, 443-445: 443.

${ }^{23}$ De Lubac zitiert hier einen Abschnitt aus einer geheimen Broschüre, die während des Konzils im Umlauf war und ihm eher aus Versehen in die Hände kam: „Unter Mutation verstehen wir, daß die Kirche sich [...] einen neuen Humanismus aneignet, der in einer und derselben offenen Synthese den ganzen eigenständigen Beitrag der großen religiösen und metaphysischen Weisheitslehren des Ostens und des Westens sowie sämtlicher Vorstöße der Natur- und Humanwissenschaften aufeinander abstimmt" (H. de Lubac, Annexe [b] [wie Anm. 22], 444). Dies sei eine radikale Umwälzung, die sich von dem dogmatischen Denken löst und dem Dialog stellt, „wenn die Kirche, im Vollzug ihrer eigenen Mutation, wahrhaft zur universellen Kirche wird“ (ebd.). 
„Eben las ich den so interessanten Bericht [...] über den Vortrag von Dr. Joseph Ratzinger am Katholikentag. [...] Und wenn sie es mir gestatten, füge ich noch hinzu: dieser Text von Dr. Ratzinger enthält das Modell einer kraftvollen Kurskorrektur, die im echten Geist des Konzils und des wahren aggiornamento dringend vorzunehmen ist" ". ${ }^{24}$ Um zu de Lubacs Aggiornamento-Verständnis vorzudringen, müssen wir also die Schrift Ratzingers befragen, der er sich anschließt. ${ }^{25}$ Mit Ratzingers Unterscheidung und Darlegung der Widersprüchlichkeit im Aggiornamento-Verständnis lässt sich genau an jene Tendenzen anknüpfen, die wir in unserem musikästhetischen Exkurs skizziert haben: auf der einen Seite die Verteidiger des antimodernistischen, restaurativ gestimmten Katholizismus von der Prägung Pius IX. (=historische Aufführungspraxis), für die Erneuerung Zerstörung der Kirche bedeutet, und auf der anderen Seite diejenigen, die sich dem Durschnittsbewusstsein von heute anpassen und „alles Ärgerliche und Wunderliche“, was sich dem entzieht, über Bord werfen wollen (= Mutation / kompositorische Neuinterpretation), dazwischen jene „zwischen den beiden Mühlsteinen [...], die mitgekämpft und mitgelitten haben, daß Erneuerung zustande komme" ${ }^{26}$ zu denen er sich selbst zählt und zu denen sich auch de Lubac zählt. ${ }^{27}$ Den Kern wahrer christlicher Erneuerung, die das Konzil vor Augen hatte, sieht Ratzinger in der Erneuerung des Christlichen, die das Christliche nicht durch et-

${ }^{24}$ H. de Lubac, Annexe [c] Brief vom 1. Dezember 1965, in: ders., Meine Schriften im Rückblick. Mit einem Vorwort von ErzbischofChristoph Schönborn (Theologia Romanica 21), Einsiedeln 1996, 446-448: 446f.

25 Ratzinger hatte sich dazu schon ein Jahr vor dem Bamberger Katholikentag in einem vor der katholischen Studentengemeinde in Münster am 18. Juni 1965 gehaltenen Vortrag geäußert, was er in seinem Referat zum Katholikentag wieder aufgenommen hatte, vgl. dazu die Anm. zu J. Ratzinger, Was heißt die Erneuerung der Kirche?, in: ders., Das neue Volk Gottes. Entwürfe zur Ekklesiologie, Patmos 1969, 267-281: 267. Schon der Beginn des Aufsatzes lässt aufhorchen bezüglich eines Aggiornamento-Wandels: „Die freudige Erregung, die der Gedanke des aggiornamento hervorrief, ist längst verebbt. Was als ein charismatisches Pfingsten begonnen hatte, ist zusehends in den Alltag eingetreten und findet sich vor die Mühseligkeiten dieses Alltags, vor seine Widersprüchlichkeiten gestellt" (ebd.).

26 J. Ratzinger, Was heißt Erneuerung (wie Anm. 25), 267.

${ }^{27}$ „Es ist das Schicksal aller Erneuerung auf dem Höhepunkt ihrer selbst, sich klären zu müssen und die Geister zu scheiden zwischen denen, die den christlichen Skandal als solchen wegleugnen wollen unter dem Vorwand, den Skandal der Christen zu beseitigen, und denjenigen, die aus der Lauterkeit ihres Glaubens heraus den wahren christlichen Skandal freilegen wollen, den das falsche Skandalum der Christenheit verdeckt" (J. Ratzinger, Was heißt Erneuerung [wie Anm. 25], 268), vgl. dazu auch die folgenden Ausführungen. 
was anderes ersetzt sehen will. ${ }^{28}$ Für ihn bildet dabei die innere Struktur des Christlichen den Bezugspunkt der Reform und nicht die Frage, was die modernen Zeiten verlangen. Das bedeutet für ihn, dass der Glaube nicht dem Geschmack des Menschen angepasst werden dürfe, sondern der Geschmack des Menschen durch den Glauben auf das wahrhaft Menschliche hingeführt werden müsse. Von dieser grundsätzlichen Frage kommt Ratzinger zum kritischen Punkt der Reform der Kirche. Der damalige persönliche Konzilsberater von Kardinal Frings sieht in Bezug auf die dem II. Vatikanischen Konzil vorausgegangene Zeit eine Verschiebung des Christlichen und einen Rückfall vom Neuen ins Alte Lied ${ }^{29}$ womit das Christliche verengt (z.B. in den Syllabi Pius IX. und X.) und die moderne Kultur verurteilt wurde. Der Kirche sei es also nicht daran gelegen gewesen, ins Heute zu gelangen, sondern im Gestern zu verharren. Aus Angst vor dem Neuen vermauerte man sich im Alten. Diese Hilflosigkeit suchte Zuflucht in der unerbitterlichen Strenge der Konservierung. Vom anderen Extrem war schon die Rede, nämlich dem Missverständnis, den Glauben so zu aggiornieren, dass man alles aus ihm entfernt, was nicht mehr dem Zeitgeist entspricht. In einem solchen Fall hält es Ratzinger für ehrlicher, direkt zu bekennen, dass man dem Christentum nichts mehr abgewinnen könne, ,anstatt aus historischen Texten Existentialien herauszuholen, die leichter ohne sie zu haben“"30 wären. Es könne für den Christen nicht genügen, sich mit den Erfordernissen und Gegebenheiten der Welt vertraut zu machen, der Christ muss vor allem mit Gott vertraut werden. Er schließt mit dem, was dem Konzil wichtig gewesen sei, dass nämlich Erneuerung Vereinfachung bedeute. Nicht jene Einfachheit der

28 Damit ist für ihn eine voraus gelagerte Frage verbunden, was denn der Kern des Christlichen sei, der nicht durch etwas anderes, scheinbar zeitgemäßeres abgelöst werden dürfe, das sich christlicher Elemente bediene, sich aber nicht an das Christliche binde. Im Grunde sei das Christentum selbst schon eine Reform gewesen, in dem es das Alte Testament erneuert habe: „Christentum ist «Neues» Testament von seinem Wesen her, die immerwährende Erneuerung vom alten Menschen her zum neuen Menschen hin, vom Alten Bund auf den Neuen zu" (J. Ratzinger, Was heißt Erneuerung [wie Anm. 25], 271). Auf einer bestimmten Ebene existiere gar nicht das immer schon sicher Bestehende, sondern Christentum sei immerwährende Neuheit, beständiger Übergang vom Alten zum Neuen Bund.

${ }_{29}$ Vgl. hierzu die bei J. Ratzinger, Was heißt Erneuerung (wie Anm. 25), 272, der hier die Psalmenauslegung aufgegriffen hat von Augustinus, in Ps. 95,2, in: CCSL 39, 1343.

30 J. Ratzinger, Was heißt Erneuerung [wie Anm. 25], 280. 
Bequemlichkeit, sondern die Einfachheit des Ursprünglichen, die der wahre Reichtum ist. ${ }^{31}$

Überspringen wir die folgenden Jahre und wenden uns direkt der jüngsten Vergangenheit und ihrer Aggiornamento-Rezeption zu, mit der uns wiederum beide Extreme begegnen werden, die sowohl unseren musikästhetischen Exkurs als auch die Reflexionen Ratzingers geprägt hatten.

\subsection{Aggiornamento in der nachkonziliaren Diskussion \\ - Schlaglichter aus der jüngsten Vergangenheit}

\subsubsection{Norbert Mette (2006)}

Im pastoraltheologischen Diskurs der Gegenwart beruft man sich selbstverständlich auf Papst, der den „Sprung nach vorwärts“32 gewagt habe. Es gelte, zeitgenössisch verständlich die Bedeutung des Christlichen heute zu vermitteln. Das Heute wird für Norbert Mette zum locus theologicus, zum Ort der theologischen Reflexion und Wahrheitsfindung. „Das hat zur Folge, dass es nicht länger die eigenen Vorstellungen sind, die den Weg der Kirche bestimmen, sondern die Menschen mit ihrer Freude und Hoffnung, mit ihrer Trauer und ihren Ängsten " ${ }^{33}$ Wenn für den Menschen von Heute die landläufige kirchliche Redeweise von Gott sinnlos geworden sei, müsse man die Vermittlung ändern, dem Menschen in seiner Lebenssituation zuhören und ihm seine Situation entschlüsseln, indem man auf biblische und nach-biblische Geschichten zurückgreife, in denen sich Menschen in einer ähnlichen Situation befunden haben. Beim Aggiornamento gehe es also darum, das Vergange hervorzuholen, damit ein neues Licht auf das Heute fällt. Das bedeutet für Mette auch ein

31 „Erneuerung ist Vereinfachung nicht im Sinne des Abschneidens oder Zerkleinerns, sondern im Sinne des Einfachwerdens, der Hinwendung zu jener wahren Einfachheit, die das Geheimnis des Lebendigen ist. Sie ist Hinkehr zu jener Einfachheit, die im letzten ein Echo ist der Einfachheit des einen Gottes. In diesem Sinne einfach zu werden - das wäre die eigentliche Erneuerung für uns Christen, für jeden einzelnen von uns und für die ganze Kirche" (J. Ratzinger, Was heißt Erneuerung [wie Anm. 25], 281).

32 Vgl. dazu und im Folgenden N. Mette, Aggiornamento der katholischen Kirche - überholt oder unerledigt?, in: „Diakonia 37“ (2006) 26-30: 27 mit dem Zitat aus Johannes XXIII., Gaudet Mater Ecclesia (wie Anm. 14), Nr. 15.

33 N. Mette, Aggiornamento (wie Anm. 32), 28. 
neues Verhältnis zur Tradition. Er plädiert ausdrücklich für eine ständige Fortschreibung der Tradition, ${ }^{34}$ mit der sich die Kirche auf die kreative Suche nach neuen, noch zu entdeckenden Landschaften machen solle. „Wir leben in einem Zeitalter der Suche: Die Menschen suchen nach verlässlichen Orientierungen für ihr Leben, nach Freiheit und Individualität, nach Geborgenheit, Gerechtigkeit und Solidarität [...]. Immer weniger können sie dabei auf einfach vorgegebene soziale Muster und Traditionen zurückgreifen. Sie müssen diese neu finden und in einen stimmigen Lebensentwurf integrieren " ${ }^{35}$ Ohne dass dies Mette noch weiter ausführt, sind mit seiner Standortbestimmung des Aggiornamento implizit verschiedene Ergebnisse und Integrationsmodelle bei einer völlig offenen Suche vorgezeichnet. Seine Konzeption der Verheutigung zielt nicht mehr in erster Linie auf Christus und seine Botschaft, der sich von dem Menschen finden lässt und von dem her dem Menschen der Sinn seines Lebens aufleuchtet. Vielmehr soll sich der suchende Mensch zu allererst selbst definieren und finden, in einer Ausdrucksvielfalt und ohne Angst, die nur lähmend wirkt auf das eigene Denken, Fühlen und Handeln. Hierin trifft sich, nur nebenbei bemerkt, Mettes Menschenbild mit dem von Zenders komponierter Interpretation der Schubert'schen Winterreise. Schon in Schuberts Original steht der Mensch als Fremder da, als Wanderer, getrieben von einer Sehnsucht, die ihn heimatlos macht. Zenders kompositorische Neuinterpretation ruft genau jenen idealtypischen spirituellen Wanderer der Spätmoderne auf den Plan, dessen Wesensmerkmal nach Martin Engelbrecht die „Gewissheit“ ist, „über alle herkömmlichen (Selbst-) abgrenzungen religiöser Institutionen und Traditionen hinaus suchen zu ,dürfen', was für einen selbst wichtig ist - die vom Göttlichen zugesprochene Ermächtigung zur religiös-sozialen, Selbstermächtigung'“ . ${ }^{36}$ Auch wenn

\footnotetext{
34 Vgl. N. Mette, Aggiornamento (wie Anm. 32), 29 mit Rekurs auf GS 91.

35 N. Mette, Aggiornamento (wie Anm. 32), 30.

36 M. Engelbrecht, Die Spiritualität der Wanderer, in: C. Bochinger; ders.; W. Gebhardt (Hg.), Die unsichtbare Religion in der sichtbaren Religion - Formen spiritueller Orientierung in der religiösen Gegenwartskultur (Religionswissenschaft heute 3), Stuttgart 2009, 35-81: 35, darin weiter: „Wanderer zu sein bedeutet in Praxis wie Theorie die stille, unspektakuläre Auflehnung gegen jegliche religiöse Geschlossenheitsansprüche im Namen der gott ge ge be ne n Freiheit des religiösen Einzelmenschen“; ausführlich dazu auch meine Ausführungen am Ende des Heilig-Geist-Bild des Wanderführers zum „spirituellen Wanderer“ in M. C. Hastetter, Pneumatologische Bildpastoral. Neue Zugänge zur Seelsorge mit Heilig-Geist-Bildern, Regensburg 2011, 742-747; vgl. zudem die erhellende
} 
Mette bestreitet, dass Aggiornamento die Anpassung an den Zeitgeist verfolge, hat er mit seiner eigenen Bestimmung von Aggiornamento als einem „Ortswechsel“37 den ursprünglichen Kern des AggiornamentoGedankens eher in Richtung einer kompositorischen Neuinterpretation weiterentwickelt.

\subsubsection{Brunero Gherardini (2010)}

Eine geradezu gegenteilige Position, die ebenfalls schon in der unmittelbaren Nachkonzilszeit vorhanden war, nimmt Brunero Gherardini in seiner 2010 erschienenen kritischen Studie in essayistischer Form über das Zweite Vatikanische Konzil ein. Statt „Christus dem modernen Menschen, seiner Mentalität, der Kultur der neuen Zeit nahezubringen“, sei man dazu übergegangen, so sein Vorwurf, „die Wahrheit aller Zeiten und für alle Zeiten der neuen und ständig wechselnden Witterung des kulturellen Klimas anzupassen" ${ }^{38}$ Mit Gherardini wird eine neue Antimodernismuswelle heraufbeschworen, die für rein historische Aufführungspraxis plädiert. Er wirft dem Konzil eine modernisierende Aggiornamento-Mentalität vor, die in vielen einzelnen Aggiornamenti (angefangen von der juridischen Struktur der Kirche bis hin zur Liturgie) Gestalt angenommen habe. Man wollte die Kirche [...] aus der ,tödlichen' Umklammerung einer Vergangenheit reißen, welche sie - im erbarmungslosen Urteil der damaligen Zeit - gefangen hielt und zu ersticken drohte". ${ }^{39}$ Gegen den Geist des Aggiornamento

Auseinandersetzung mit Peter Sloterdijk und der Fremdheit als existentiale Kategorie in Bezug auf Zenders Neuinterpretation der Winterreise bei K. P. Liessmann, Anmerkungen zu Hans Zenders komponierter Interpretation von Franz Schuberts „Winterreise“, in: Booklet zur CD Hans Zender, Schuberts „Winterreise“. Eine komponierte Interpretation. Christoph Prégardien. Klangforum Wien. Sylvian Cambreling, Kairos Music Production 1999, 14-17.

37 N. Mette, Aggiornamento (wie Anm. 32), 30, der sich hier bezieht auf die Ausführungen von H.J. Sander, Theologischer Kommentar zur Pastoralkonstitution über Kirche und Welt von heute „Gaudium et spes“, in: P. Hünermann; B. J. Hilberath (Hg.), Herders Theologischer Kommentar zum Zweiten Vatikanischen Konzil. Bd. 4, Freiburg 2005, 585-869: insb. 588-591. Nur nebenbei angemerkt kommt Sander im gesamten Kommentar zu Gaudium et Spes ohne die Verwendung der Begrifflichkeit von "Aggiornamento“ oder „Verheutigung“ aus. An ihre Stelle treten die Paradigmen „Ortsbestimmung“ und „Ortswechsel“, vgl. ebd., 585, 586, 588 u.a.

38 B. Gherardini, Das Zweite Vatikanische Konzil. Ein ausstehender Diskurs, Übersetzt von Claudia Barthold, Mülheim-Mosel 2010, 38.

39 Ebd. (wie Anm. 38), 41. 
polemisiert Gherardini mit kriegerischem Vokabular ${ }^{40}$ und entlarvt ihn als "ein traurige[s] und bittere[s] ,Modernisierungswerk'“ - ein „De profundis auf die Vergangenheit und ein Hymnus auf die Sonne der Zukunft". ${ }^{41}$ Wenn wir uns zurückerinnern, hatte aus der Erfahrung der Musikgeschichte selbst Harnoncourt die historische Schau für eine kulturell vitale Zeit als absolut wesensfremd eingestuft. ${ }^{42}$

\subsubsection{Zwischenbilanz}

Halten wir bis hierher fest: Die Auseinandersetzung um das recht verstandene Aggiornamento, das in seiner Realisierung der Praxis je neu aufgetragen ist und sich letztlich in der Praxis entscheidet, ist bis heute nicht zur Ruhe gekommen. Es scheint fast, als sei ein neuer Kampf um die Hermeneutik der Verheutigung entbrannt, dessen tiefere Ursache Karl Lehmann in seinem vielrezipierten Eröffnungsreferat „Neuen Zeichen der Zeit" zur Vollversammlung der Deutschen Bischofskonferenz 2005 zu erörtern versucht hat. Nach einer eingehenden Analyse der Krisenerscheinungen der Gegenwart widmet er sich dem „Gleichgewicht zwischen Wandel und Beständigkeit " ${ }^{43}$ worin er die Unausgeglichenheit in der Kirche heute kritisiert, die sich immer nur auf das Momentane und Gegenwärtige beschränkt. „Darum“, so Lehmann, „leben wir oft auch in der Kirche viel zu heutig. Es

40 „Abgesehen von einer Minderheit, die nicht ohne Pfeile vom eigenen Bogen, dem Sturmangriff der Neuerer gegen die Festung der traditionellen Werte Widerstand leistete, marschierte die große Mehrheit mit raschem Schritt in die Richtung des «tiefgreifenden Aggiornamento». [...] Es war offensichtlich, dass im Inneren der Konzilsaula die alten modernistischen Vorwürfe wieder auftauchten, denen Pius X. zu seiner Zeit die Stirn geboten hatte, die aber nicht bezwungen waren. Man ertrug den Antimodernismuseid nicht, den Pius X. für alle Kirchenmänner als obligatorisch vorgeschrieben hatte, und man wünschte seine Verbannung auf ewig“ (ebd. [wie Anm. 38], 42).

${ }^{41}$ Ebd. (wie Anm. 38), 46. Letztlich führt Gherardini in seiner Argumentation jene Denkströmung weiter, die Aggiornamento als das ,Zentralmotiv einer mutwilligen Anpassung der Kirche an den Zeitgeist („Verweltlichung“) sowie als Ausverkauf und Preisgabe wesentlicher Gehalte des Glaubens und der Kirche interpretiert" (M. Bredeck, Zweite Vatikanum [wie Anm. 12], 60 mit Rekurs auf die Aufarbeitung der Levebvre-Bewegung bei B. Schoppelreich, Zeichen und Zeugnis. Zum sakramentalen Verständnis kirchlicher Tradition [Studien zur Traditionstheorie 3], Münster 2001, 132).

42 Vgl. N. Harnoncourt, Musik als Klangrede (wie Anm. 9), 14.

43 K. Lehmann, Neue Zeichen der Zeit. Unterscheidungskriterien zur Diagnose der Situation der Kirche in der Gesellschaft und zum kirchlichen Handeln heute. Eröffnungsreferat von Karl Kardinal Lehmann bei der Herbst-Vollversammlung der Deutschen Bischofskonferenz in Fulda (19. September 2005) (Der Vorsitzende der Deutschen Bischofskonferenz 26), Bonn 2006, 25. 
geht nicht mehr, wie in der Konzilszeit, um das ,aggiornamento', d.h. um die lebendige Vergegenwärtigung der geschichtlichen Überlieferung in das Heute hinein. Uns fehlt der lange Atem. [...] Wir treten oft hektisch auf der Stelle und befinden uns auf der Suche nach der Gegenwart oft in einem flüchtigen Niemandsland. Es ist dann kein Wunder, dass wir im hohen Maß den gesellschaftlichen Strömungen ausgeliefert sind, uns an ihre Trends verlieren und keinen eigenen Ort mehr gewinnen, der uns Stand gibt, standhalten lässt und uns zum Widerstand befähigt" “. ${ }^{44}$

Lehmanns Einschätzung der Problematik in Hinblick auf ein Übergewicht an Verheutigung deckt sich mit der von Zenders interpretatorischer Neukomposition der Winterreise. Beim ersten Höreindruck fasziniert und begeistert die neue Klangfarbe, der Wechsel, die Mutation, mit der dieser ästhetische Transfer einhergeht. Aber im objektiven Vergleich von Original und neuem Original wirkt Zenders Fassung nicht nur länger und zuweilen auch langatmiger, sondern überragt Schuberts Winterreise zeitlich um ganze 20 Minuten. ${ }^{45}$ Zudem ist die Orchesterbesetzung der kompositorischen Interpretation Winterreise sehr viel aufwendiger und nicht zuletzt in ihrer Aufführung um ein Vielfaches teurer. Ein mir bekannter Kirchenmusiker und Komponist, der bei der Uraufführung Zenders Neukomposition der Winterreise anwesend war, hatte den Eindruck: „Am Ende von Zenders interpretatorischer Komposition ist mir erst die Schönheit der Winterreise im Original aufgegangen: ihre Einfachheit, ihr Frische und diese Tiefe im Ausdruck und das alles mit den sparsamen Mitteln eines einzigen Klaviers und nur einer Stimme“. ${ }^{46}$ Ähnliche Erfahrungen lassen sich auch bei verschiedentlichen pastoralen Neukonzeptionen feststellen: Wie viel Aufwand wird betrieben, um immer neue Konzepte zu erarbeiten, um die Kirche attraktiver zu machen, um aus dem Alten Neues werden zu lassen, ohne sich der Folgen bewusst zu werden, die auch schon in Zenders Neuinterpretation hervorgetreten sind: Langatmigkeit, hoher

$44 \quad$ Ebd. (wie Anm. 43), 26.

45 H. Zenders Winterreise dauert 93:49 Minuten (Aufnahme vgl. Anm. 3), Schuberts Winterreise in einer Einspielung mit dem gleichen Sänger hingegen nur 73:58 Minuten, vgl. F. Schubert, Winterreise op. 89 D 911 zu Gedichten von Wilhelm Müller. Christoph Prégardien, Tenor. Andreas Staier, Klavier, Westdeutscher Rundfunk Köln, Teledec Classics International, Hamburg 1997.

46 So Stephan Heuberger im Gespräch zu mir im Jahr 2011. 
Kosten-, Organisations- und Zeitaufwand, Entfremdung vom Original statt einfach(er)e Seelsorge. ${ }^{47}$

Hier könnten wir unsere Ausführungen nun schließen. Aber wäre damit schon alles zur pastoralen Bedeutung des Aggiornamento gesagt? Genügt es, die kirchlichen Lebensvollzüge in Rückbindung an die Tradition an das Heute anzupassen? Genügt es, Aggiornamento in der Pastoral objektiv als Synthese von Treue und Dynamik, zu verstehen?

\section{Das Neue Lied - das Lied neu singen}

Bislang bewegten sich unsere Ausführungen zum Aggiornamento weitgehend auf der Ebene einer von der eigenen Person distanzierten objektiven Zeit- und Gesellschaftskritik. Ratzinger hingegen hatte bereits 1965 beim Aggiornamento neben dem objektiven pastoralen Moment in Anlehnung an Augustinus noch eine existentielle seelsorgliche Komponente herausgehoben. Augustinus sei es bei der Verheutigung des alten Liedes um das Wesentliche des eigenen Christseins, also um etwas Existentielles gegangen: „Was immer du singst aus der Selbstsucht heraus, es bleibt das alte Lied, selbst wenn man dabei die Worte des neuen hört". ${ }^{48}$ - Für Ratzinger will das sagen: „Die eigentliche Erneuerung liegt nicht in neuen Buchstaben; ob sie gelingt, hängt davon ab, wie weit die neuen Formen zum Medium jenes wesentlichen Schrittes werden, der im Übergang vom alten zum neuen Menschen besteht, von der Selbstsucht zur Liebe "49 Diese exis-

47 Das Modell der einfach(er)en Seelsorge wurde von Hubert Windisch in verschiedenen Veröffentlichungen thematisiert, vgl. dazu beispielsweise H. Windisch, Einfach(er)e Seelsorge. Das Pfarrprojekt Paroikia, in: P. Müller; ders. (Hg.), Seelsorge in der Kraft des Heiligen Geistes, Freiburg 2005, 245-258; ders., Einfach(er)e Seelsorge. Hinführung zu einem Pfarrprojekt, in: LS 58 (2007) 256-259; ders., Minima Pastoralia. Orientierungshilfen für die Seelsorge, Würzburg 2001.

48 Augustinus, in Ps. 95, 2, in: CCL 39, 1343; deutsch nach J. Ratzinger, Was heißt Erneuerung (wie Anm. 25), 272.

49 J. Ratzinger, Was heißt Erneuerung (wie Anm. 25), 272; ähnlich später in seiner Rezension zu Willams Studie, wo er seine Analyse der Aggiornamento-Elemente (Unterscheidung von Substanz und Akzidenz, keine steckendürre Reproduktion, lebendiger Saft, Lebensumstände und Talente) weiterführt: „Das aber bedeutet: Die Idee des Aggiornamento ist zunächst nicht auf die Fragen der theologischen Doktrin oder auf die Veränderung und Erneuerung der Kirche bezogen gewesen, sondern hat ihre Wurzel im Ringen um die wahre Form der Heiligkeit. Nur von diesem Zentrum 
tentielle Deutung von Aggiornamento, die zur objektiven hinzutritt, legt den Ursprung des Aggiornamento-Verständnisses bei Angelo Giuseppe Roncalli frei, den er schon Anfang 1903 in seinem Exerzitientagebuch nach der Lektüre von Alfred Loisys gerade erschienenem Werk „L'Évangile et L'Église“ (November 1902) eingeschlagen hatte. ${ }^{50}$ Willam fasst aufgrund

her kann sie sachgemäß verstanden werden - das ist die entscheidende Einsicht, die sich hier für das Verständnis des wahren Wollens von Papst Johannes ergibt“ (J. Ratzinger, Rezension zu: Franz Michel Willam, Vom jungen Angelo Roncalli [1903-1907] zum Papst Johannes XXIII. [1958-1963], Innsbruck 1967, in: ThQ 148 [1968] 236-241: 238). Wieder aufgegriffen hat Ratzinger diese existentielle Deutung von Aggiornamento dann im Kommentar zu Kap. 1 des ersten Teils (Die Würde der menschlichen Person). Art. 11-22 der Pastoralkonstitution über die Kirche in der Welt von heute - Gaudium et spes, in: ${ }^{2}$ LThK. Ergänzungsband III (1968) 313-354: 314, worin es um die Unterscheidung (und nicht mehr wie im Vorläufertext das Aufspüren) der Zeichen der Zeit geht. Diese zur christologischen und chronologischen Sicht mit ihrer Verpflichtung auf Kontinuität und Identität hinzutretende pneumatologische Perspektive rücke den kairologischen Aspekt in den Vordergrund, in dem sich „das Werk des Herrn als gegenwärtiges vollziehen muß“ (ebd.). Die Unterscheidung der Geister als ein Unterscheiden zwischen Zeitgeist und dem Augenblick des Heiligen Geistes führt Ratzinger zur eigentlichen Bestimmung von Aggiornamento, „damit nicht unter dem Schein des Gehorsams gegenüber dem Pneuma sich die Unterwerfung unter das Diktat der Mode und der Abfall vom Herrn vollzieht. Damit wird die notwendige Verbindung von Heiligkeit und Aggiornamento sichtbar: als Eingehen auf den Kairos muß es zugleich Dia-krisis seiner Geister aus dem Stehen im einen Geist des Herrn sein“ (ebd.). Eine Weiterführung dieses Gedankengangs findet sich bei ihm dann zwanzig Jahre später in seiner „Einführung in das Christentum“: „Brauchen wir nur zum Aggiornamento zu greifen, uns abzuschminken und uns in das Zivil einer säkularen Sprache oder eines religionslosen Christentums zu stecken, damit alles in Ordnung sei? Genügt der geistige Kostümwechsel, damit die Menschen freudig herbeilaufen und mithelfen, den Brand zu löschen, von dem der Theologe behauptet, daß es ihn gebe und daß er unser aller Gefahr sei? [...] Wenn indes der, der den Glauben zu verkündigen versucht, selbstkritisch genug ist, wird er bald bemerken, daß es nicht nur um eine Form, um eine Krise der Gewänder geht, in denen die Theologie einherschreitet. In der Fremdheit des theologischen Unterfangens den Menschen unser Zeit gegenüber wird der, der seine Sache ernstnimmt, nicht nur die Schwierigkeit der Dolmetschung, sondern auch die Ungeborgenheit seines eigenen Glaubens, die bedrängende Macht des Unglaubens inmitten des eigenen Glaubenwollens erfahren“ (ders., Einführung in das Christentum. Vorlesungen über das Apostolische Glaubensbekenntnis, München 1968, 18f.).

50 Dieser Zusammenhang ist aufgearbeitet worden von F. M. Willam, Vom jungen Angelo (wie Anm. 13), 10-34; vgl. dazu auch A. Loisy, L'Évangile et L'Église, Frankfurt 1973 (Unveränderter Nachdruck der vierten Auflage von 1908 [Erstauflage 1902]), 153-155, 166, hier zum Beispiel wörtlich: „la loi de la vie, laquelle es tun mouvement et un effort continuel d'adaptation à des conditions perpétuellement variables et nouvelles. Le christianisme n'a pas échappé à cette loi, et il ne faut pas le blâmer de s'y être soumis“ (ebd., 154) oder „la puissance d'adaptation“ (ebd., 166), die er dann ausführt als einen Umstand, dass das Evangelium nicht eine absolute und abstrakte Lehre für alle Zeiten sei, sondern in jeder Zeit ununterbrochen angepasst werden müsse; dies bedeute, dass sich auch die Kirche fortwährend den Bedingungen der neuen Zeiten anpassen müsse (vgl. ebd., 166). 
der Auswertung des "Giornale“ seine Leitidee wie folgt zusammen: „Jedem Menschen ist es aufgegeben, die Grundsätze des Glaubens auf die ihm eigenen persönlichen Anlagen und Lebensbedingungen hin anzuwenden ". ${ }^{51}$ In der Roncalli-Forschung herrscht Einmütigkeit darüber, dass beim späteren Papst Johannes XXIII. Aggiornamento dem individuell persönlichen Streben nach Heiligkeit entspringt - einer Kunst, die dann nach Außen wirksam wird in der Auseinandersetzung mit der gegenwärtigen Zeit. ${ }^{52}$ Aggiornamento in seiner existentiellen pastoralen Ausformung ließe sich somit auf die Kurzformel bringen: die Kunst Heiligkeit unter den Bedingungen der Gegenwart. ${ }^{53}$

Wenn man diesen existentiellen Verstehensansatz zum Aggiornamento bei Angelo Roncalli nun rückbindet an unseren Ausgangspunkt, hieße das auf die Musik übertragen: Für die Lieder der Winterreise genügt es nicht nur, zeitgemäße Einführungstexte zu neuen Aufführungen und Einspielungen $\mathrm{zu}$ erstellen, um sie dem Hörer von Heute nahezubringen (=objektive Komponente); vielmehr sind sie auch selbst zu singen und zu spielen (= existentielle Komponente), um auf diese Weise an ihrer Verheutigung mitzuwirken ${ }^{54}$ Aggiornamento im Sinne von Angelo Roncallis existenti-

51 F. M. Willam, Vomjungen Angelo (wie Anm. 13), 27. Ratzinger hat die Stelle aus dem Tagebuch Johannes XXIII., wo er sich mit der steckendürren Reproduktion auseinandersetzt (vgl. Anm. 19) später in einer Predigt zu Franz von Sales wieder aufgegriffen und die Idee des ,neu, aufmeine Art, Früchte tragen“, weiter entfaltet: „Darum geht es: Frucht tragen - jeder auf seine Art. Gott hat einen reichen Garten geschaffen und jedem seine Weise der Heiligkeit gegeben, in der Gottes Blumen blühen und Früchte reifen. Nicht wie in steckendürrer Reproduktion nachahmen, sondern den eigentlichen sugo vitale, den Lebenssaft des Evangeliums, die eigentliche tiefere Essenz des Heiligen den Heiligen ablauschen, damit sie in uns neuer Lebenssaft wird, der auf seine Weise Frucht trägt und Gottes Anruf erfüllt, Gottes Möglichkeiten in der Schöpfung zum Blühen und Reifen bringt" (J. Ratzinger, Heiligenpredigten, Herausgegeben von Stephan Otto Horn, Unter Mitarbeit von Gabriele Besold, München 1997, 24).

52 Auf das Moment der Kunst (ars) im Gegensatz zur Wissenschaft (scientia) beim AggiornamentoVerständnis Roncallis hat schon Willam hingewiesen, vgl. F. M. Willam, Vomjungen Angelo (wie Anm. 13), 148f.; vgl. hierzu auch die Entfaltung bei J. Ratzinger, Rezension Willam (wie Anm. 49), 238.

53 Vgl. hierzu M. Bredeck, Zweite Vatikanum (wie Anm. 12), 159. Diese Kurzformel schließt im Grunde alle vier Aggiornamento-Ebenen mit ein, die Bredeck herausgearbeitet hat: (1) Aggiornamento als intensives Streben nach Heiligkeit, (2) Aggiornamento als Dienstbereitschaft füreinander, (3) Aggiornamento als Zuwendung zum Mitmenschen, (4) Aggiornamento als Bewusstsein für die Herausforderungen der Gegenwart, vgl. ebd., 261.

${ }^{54}$ Zu bedenken wäre hierzu noch einmal in Bezug auf Schuberts Winterreise, dass jede neue Einspielung bereits eine Neuinterpretation des Werkes ist durch die konkreten Ausführenden 
ellen Verstehenshorizont schafft Raum für die „existentielle Wucht des Originals" (Zender) - auf der Seite der Seelsorge. Damit gestaltet sich eine an der Kunst des Aggiornamento ausgerichtete Pastoral im Sinne eines mehrschichtigen Prozesses: Für die inhaltlichen oder vertikalen Ebene wirkt die Synthese von Treue und Dynamik bestimmten, die sich an ihren Rändern von den beiden Extremen des Musealen und der Mutation abgrenzt. Auf der Handlungsebene vereinigen sich eine objektive, also von außen wahrnehmende und reflektierende Haltung mit einer existentiellen Offenheit auf Christus hin im hic et nunc. Erst wenn alle vier Komponenten (Treue, Dynamik, objektive Reflexion und existentielle Aneignung) zusammenwirken, kann man vom pastoralen Aggiornamento in seiner vollen Bedeutungstiefe sprechen. Anhand dieser vier Felder ist der Praxis der Seelsorge somit ein Instrumentar zur Hand gegeben, pastorales Handeln auf Aggiornamento hin überprüfen.

Fazit: Das neue Lied zu singen, oder vielmehr das Lied neu zu singen bleibt in der Pastoral eine hohe Kunst. Die Worte von Johannes XXIII. kurz vor Konzilsbeginn in einer Ansprache an Pädagogen stellen so etwas wie die pastorale Gesangsanleitung zu diesem neuen Lied dar: keine Kompromisse mit dem Zeitgeist einzugehen, sondern, im Gegenteil, im kirchlichen Handeln den Erfordernissen der Moderne zu entsprechen und dabei immer tiefer die Gestalt Jesu, des Gekreuzigten, zu assimilieren. ${ }^{55}$

Lassen Sie mich mit einem Gedanken von Augustinus schließen: Wer das neue Lied singt, baut auf, denn singen heißt aufbauen, solange man nicht das alte Lied der Leidenschaften singt, sondern das neue Lied, das „,aritas Dei “ heißt. ${ }^{56}$ Wird das neue Lied der Liebe gesungen, so wird das Haus, das

(in der Fachsprache die „Interpreten“), die ihm eine unverkennbare und einzigartige Färbung verleihen.

55, ,N]on vuole dire accertare compromessi con lo spirito secolare [...]; al contrario l'adeguamento moderno alle esigenze dei tempi dovrà risolversi in una più profonda assimilazione alle personalità di Gesù Crocifisso" (Johannes XXIII., Udienza per i participanti al corso di aggiornamento pedagogico, in: DMC IV, 515).

56 Vgl. hierzu Augustinus, in Ps. XCV, 2, in: CCSL 39, 1343, hier im lateinischen Original: „Si canticum nouum cantat omnis terra; sic adeificatur, cum cantat: ipsum cantare, aedificare est; sed si non uetus cantet. Vetus cantat cupiditas carnis: nouum cnatat cartitas Dei [...]. Omnis ergi terra cantat canticum noum; ibi aedificatur domus. Omnis ergo terra domus Dei. Si omnis terra domus Dei, qui non haeret omni terrae, ruina est, non domus; ruina illa vuetus, cuius umbram habebat templum illud uetus. Ibib enim diruebatur uetustas, ut nouitas aedificaretur“. 
die Kirche ist - und wir könnten ergänzen: so werden unsere Pfarreien, kirchliche Bewegungen, Gemeinschaften und Familien, die Gremien, Räte, Komissionen, Ausschüsse und kirchlichen Sozialeinrichtungen -, aufgebaut, das mit dem alten Lied in Ruinen liegen würde. Denn die Neuheit der Liebe baut auf (nouitas adificaretur).

\section{Summary}

To Sing the New Song... About the Art of Aggiornamento

The postmodern comprehension of aggiornamento, which continues the tradition in a certain artistic freedom from the origin, is different from the well-balanced aggiornamento understanding of the Council Fathers. They intend to liberate the Christian from solidification as well as overgrowth and to focus on the essential of the Christian belief, namely Jesus Christ. In the recent time there has been additionally a historicizing tendency of aggiornamento, which looks back again at the historical performance practice and belongs to the field of it. Referring to statements of important Council fathers, aggiornamento is developed as a process with several aspects for the pastoral practice, which also includes an existential level.

Keywords: II Vatican Council, Interpretation, Tradition, Reform, Practical Theology 